\title{
Pomeron and odderon in QCD and a two dimensional conformal field theory
}

\author{
L.N. Lipatov ' \\ Deutsches Elektronen-Synchrotron-DESY, W-2000 Hamburg, FRG \\ Received 18 May 1990; revised manuscript received 29 August 1990
}

\begin{abstract}
The problem of solving the Bethe-Salpeter equations in LLA for $t$-channel partial waves corresponding to Feynman diagrams with many reggeized gluons is simplified significantly by using their conformal invariance in the impact parameter representation and the separability property of their integral kernels. In particular, for the three gluon system with the odderon quantum numbers we obtain a one dimensional integral equation.
\end{abstract}

It is known [1] that in the leading logarithmic approximation (LLA) the gluon production amplitudes at large energies $\sqrt{s}$ have the multi-Regge form and are expressed in terms of the reggeized gluon trajectory $j=1+\omega, \omega \sim g^{2}$ and the reggeon-reggeonparticle vertex $\gamma \sim g$, where $g$ is the QCD coupling constant. High order radiative corrections to these quantities and many reggeon vertices can be calculated using a dispersive approach [2].

The hadron scattering amplitudes in LLA are expressed through the solution of the Bethe-Salpeter equation for $t$-channel partial waves $f_{\omega}\left(k, k^{\prime}, q\right)$ describing the pomeron built from two reggeized gluons $[1,3]$. Further, the analogous equation for the three gluon compound state $\psi\left(k_{i}\right)(i=1,2,3)$ with the odderon quantum numbers $\left(P_{j}=C=-1\right)$ is constructed with the use of the integral kernels for pairlike gluon interactions which are proportional to the pomeron kernel [4].

It is convenient to perform the Fourier transform of the function $f_{\omega}\left(\boldsymbol{k}_{i}, \boldsymbol{k}_{\boldsymbol{i}}\right)$ depending on transverse components $\boldsymbol{k}_{i}, \boldsymbol{k}_{i} \cdot(i=1,2, \ldots, n)$ of virtual gluon momenta and to pass to the impact parameter representation $f_{\omega}\left(\boldsymbol{\rho}_{i}, \boldsymbol{\rho}_{i}\right)$ (here $\boldsymbol{\rho}_{i}$ and $\boldsymbol{\rho}_{i}$ are the transverse coordinates of the initial and final gluons in the $t$ channel). The Bethe-Salpeter equations in this rep-

' Permanent address: Leningrad Nuclear Physics Institute, Gatchina, SU-188 350 Leningrad, USSR. resentation are conformal invariant and their solutions $f_{\omega}\left(\boldsymbol{\rho}_{i}, \boldsymbol{\rho}_{i^{\prime}}\right)$ can be interpreted as the Green functions of a two dimensional euclidean field theory [5],

$f_{\omega}\left(\boldsymbol{\rho}_{i}, \boldsymbol{\rho}_{i^{\prime}}\right) \equiv\left\langle 0\left|\prod_{i} \varphi\left(\boldsymbol{\rho}_{i}\right) \prod_{i^{\prime}} \varphi\left(\boldsymbol{\rho}_{i^{\prime}}\right)\right| 0\right\rangle$.

Here the fields $\varphi\left(\rho_{i}\right), \varphi\left(\rho_{i^{\prime}}\right)$ describe virtual gluons and belong to the adjoint representation of the $\operatorname{SU}(N)$ gauge group.

By inserting in eq. (1) the unit operator expanded in the sum of projectors into the complete set of states corresponding some local operators $O_{k}\left(\rho_{0}\right)$ one can express $f_{\omega}$ in terms of simpler matrix elements $\chi\left(\boldsymbol{\rho}_{i}\right.$, $\left.\rho_{0}\right):$

$$
\begin{gathered}
f_{\omega}\left(\boldsymbol{\rho}_{i}, \boldsymbol{\rho}_{i^{\prime}}\right)=\sum_{k} \int \mathrm{d}^{2} \rho_{0} \chi_{k}\left(\boldsymbol{\rho}_{i}, \boldsymbol{\rho}_{0}\right) \chi_{k}^{*}\left(\boldsymbol{\rho}_{i^{\prime}}, \boldsymbol{\rho}_{0}\right), \\
\chi_{k}\left(\boldsymbol{\rho}_{i}, \boldsymbol{\rho}_{0}\right) \equiv\left\langle 0\left|\prod \varphi\left(\boldsymbol{\rho}_{i}\right) O_{k}\left(\boldsymbol{\rho}_{0}\right)\right| 0\right\rangle .
\end{gathered}
$$

The $n$ gluon wave function $\chi$ satisfies in LLA the following homogenous equation [3-6]:

$$
\begin{aligned}
& \omega \chi\left(\boldsymbol{\rho}_{i}, \boldsymbol{\rho}_{0}\right) \\
& =\frac{g^{2}}{8 \pi^{2}} \sum_{r<s}\left(-T_{r}^{a} T_{s}^{a}\right) K\left(\boldsymbol{\rho}_{r}, \boldsymbol{\rho}_{s}\right) \chi\left(\boldsymbol{\rho}_{i}, \boldsymbol{\rho}_{0}\right),
\end{aligned}
$$

where the colour group generators $T_{n}, T_{s}$ and the integral operators $K\left(\boldsymbol{\rho}_{n}, \boldsymbol{\rho}_{s}\right)$ act as the variables of the $r$ th and sth gluon.

The eigenvalues and the eigenfunctions of eq. (3) 
determine the position of the singularities of the partial waves $f_{\omega}$ in LLA and their discontinuities on these singularities. In the complex coordinates $\rho_{i}, \rho_{i}^{*}$ for impact parameters $\rho_{i}$ the operator $K$ in eq. (3) has the surprisingly simple separable structure [6]

$$
\begin{aligned}
& K\left(\boldsymbol{\rho}_{r}, \boldsymbol{\rho}_{s}\right)=k\left(M_{r s}^{2}\right)+k\left(M_{r s}^{* 2}\right), \\
& k\left(M_{r s}^{2}\right)=\sum_{l=0}^{\infty}\left(\frac{2 l+1}{l(l+1)-M_{r s}^{2}}-\frac{2}{l+1}\right) .
\end{aligned}
$$

Here

$$
\begin{aligned}
M_{r s}^{2} & \equiv\left(M_{r s}^{0}\right)^{2}-\frac{1}{2}\left(M_{r s}^{+} M_{r s}^{-}+M_{r s}^{-} M_{r s}^{+}\right)=-\rho_{r s}^{2} \partial_{r} \partial_{s}, \\
\rho_{r s} & \equiv \rho_{r}-\rho_{s}, \quad \partial_{r} \equiv \frac{\partial}{\partial \rho_{r}},
\end{aligned}
$$

is the Casimir operator for the Möbius group of conformal transformations and

$M_{r s}^{0}=\rho_{r} \partial_{r}+\rho_{s} \partial_{s}$,

$M_{r s}^{+}=\rho_{r}^{2} \partial_{r}+\rho_{s}^{2} \partial_{s}, \quad M_{r s}^{-}=\partial_{r}+\partial_{s}$

are their generators $\left(M_{r s}^{\nu}=M_{r}^{\nu}+M_{s}^{\nu}\right)$.

The total momentum squared $M^{2}=\left(\sum_{s} M_{s}\right)^{2}$ commutes with all $K$. Therefore $\chi$ belongs to a representation of the Möbius group:

$M^{2} \chi=m(m-1) \chi, \quad M^{* 2} \chi=\tilde{m}(\tilde{m}-1) \chi$

and the eigenvalues $\omega$ are some functions of the conformal weight $m$ and $\tilde{m}$ :

$$
\begin{aligned}
& \omega=\omega(m, \tilde{m}), \quad D=m+\tilde{m}=1+2 \mathrm{i} \nu, \\
& n=m-\tilde{m},
\end{aligned}
$$

where $D$ and $n$ are the dimension and the conformal spin of the operator $O_{k}\left(\rho_{0}\right)$ respectively.

From eqs. (4) $-(6)$ one can obtain the normalization condition for $x$.

$\|\chi\|^{2}=\int \prod_{i} \mathrm{~d}^{2} p_{i} \chi^{*} \prod_{r}\left|\partial_{r}\right|^{2} \chi$.

It is remarkable that the function $\omega(9)$ has simple analytic properties in the variables $m$ and $\tilde{m}$ : it contains poles at integer points $k=1,2, \ldots$ and $\tilde{k}=1,2, \ldots$ accordingly. To find the residues in these poles let us notice firstly that in integer $m, \tilde{m}$ the operator $O\left(\rho_{0}\right)$ can be constructed as a conformally covariant polynomial in the derivatives of the free fields $\varphi$. Therefore the matrix element (2) for $m=k, \tilde{m}=\tilde{k}$ equals

$$
\begin{aligned}
& \chi^{k, k}=\sum_{\left\{k_{i}\right\}} \sum_{\left\{k_{i}\right\}} C_{\left\{k_{i}\right\}\left\{k_{i}\right\}} \prod_{i}\left(\frac{\rho_{i 1}}{\rho_{i 0} \rho_{10}}\right)^{k_{i}} \prod_{i}\left(\frac{\rho_{i 1}^{*}}{\rho_{i 0}^{*} \rho_{i 0}^{*}}\right)^{k i}, \\
& \sum k_{i}=k, \quad \sum k_{i}=k,
\end{aligned}
$$

where the coefficients $C_{\left\{k_{i}\right\}\left\{k_{i}\right\}}$ can be calculated from a secular equation derived from eq. (3). However, in the pole approximation for $\omega(m, \tilde{m})$ it turns out that the corresponding anomalous dimension matrix is degenerate.

To show this in general, it is necessary to consider a trial function $\chi$ which is nonsingular at $\rho_{i j}=0$ for all $i$ and $j$, has the correct dimension and coincides with (11) in the limit $m \rightarrow k, m \rightarrow \tilde{k}$. But when on the RHS of eq. (3) we apply one of the operators $K\left(\rho_{r}, \rho_{s}\right)$ to this function it is possible to restrict ourselves to the simpler expression

$\chi=\left(\frac{\rho_{r s}}{\rho_{r 0} \rho_{s 0}}\right)^{m-k}\left(\frac{\rho_{r s}^{*}}{\rho_{r 0}^{*} \rho_{s 0}^{*}}\right)^{\tilde{m}-\bar{k}} \chi^{k, \kappa}$,

because $K\left(\rho_{m}, \rho_{s}\right)$ does not strengthen its singularity. Moreover, $\chi(12)$ is an eigenfunction of $K\left(\rho_{r}, \rho_{s}\right)$. Then using the same procedure for each kernel (5) on the RHS of eq. (3) we obtain its eigenvalue in the pole approximation,

$\left.\omega\right|_{m \rightarrow k, \dot{m} \rightarrow \kappa}=\frac{g^{2}}{8 \pi^{2}} \frac{n}{2} N\left(\frac{1}{k-m}+\frac{1}{\tilde{k}-\tilde{m}}\right)$,

where we used the identity $\sum_{r<s}\left(-T_{r}^{a} T_{s}^{a}\right)=\frac{1}{2} n N$. The result (13) is valid for arbitrary linear combinations of eigenfunctions (11) with $k_{i}, k_{i} \geqslant 1$, which corresponds to the matrix elements of the gauge invariant operators. The simple analytic structure of $\omega$ (13) and the large degeneracy of the anomalous dimension matrix in the pole approximation are presumably important for finding an exact solution of eq. (3).

Let us return now to the separability relation (4) for the kernel $K$. In the cases where the colour structure of $\chi$ is factorized (it takes place for $n=2,3$ and $N=\infty$ ) we can write it down due to eq. (4) in the form

$\chi\left(\boldsymbol{\rho}_{i}, \rho_{0}\right)=\sum_{r} \chi^{r}\left(\rho_{i}, \rho_{0}\right) \tilde{\chi}^{2}\left(\rho_{i}^{*}, \rho_{0}^{*}\right)$,

where $\chi^{r}$ and $\tilde{\chi}^{r}$ satisfy the simpler equations in which $K$ is substituted by $k$. The functions $\chi^{r}, \tilde{\chi}^{r}$ are analo- 
gous to "conformal blocks" of conformal field theory [7].

To construct $k\left(M_{r s}^{2}\right)$ in the form of an integral operator one can use its following representation:

$$
\begin{aligned}
& k\left(M_{r s}^{2}\right)=\int_{-\infty}^{+\infty} \mathrm{d} \tau\left(\varepsilon(\tau) \operatorname{tr} \exp \left(-\tau M_{r s}^{0}\right)\right. \\
& \left.-\frac{\exp (-|\tau|)}{1-\exp (-|\tau|)}\right),
\end{aligned}
$$

where " $t r$ " in front of the subsequent operator means the sum over its matrix elements between the states inside the irreducible representation with the highest weight $m-1\left(\chi^{(m-1)}=\rho_{r s}^{1-m}\right)$.

Expression (15) can be rewritten using the operation of the invariant integration as

$$
\begin{aligned}
& k\left(M_{r s}^{2}\right)=\frac{1}{4 \pi} \int \frac{\mathrm{d}^{3} Z}{Z_{\mu}^{2}} \mathrm{cth} \sqrt{-\frac{1}{4} Z_{\mu}^{2}} \\
& \times \varepsilon\left(Z_{0}\right) \theta\left(-Z_{\mu}^{2}\right) \exp \left(Z_{\mu} M_{\mu}^{r s}\right)-C, \\
& C=2 \int_{0}^{\infty} \mathrm{d} \tau \frac{\exp (-\tau)}{1-\exp (-\tau)}, \\
& \tau=\sqrt{-Z_{\mu}^{2}}, \quad Z_{\mu}^{2}=-Z_{0}^{2}+Z_{1}^{2}+Z_{2}^{2} .
\end{aligned}
$$

Here $Z_{\mu}$ and $M_{r s}^{\mu}$ are vectors in the three dimensional Minkowski space.

The factor $\exp \left(Z_{\mu} M_{r s}^{\mu}\right)$ carries out the arbitrary Möbius transformation of the variables $\rho_{r}, \rho_{s}$ with the group parameters $Z_{\mu}$. Therefore, eq. (16) can be presented in the form of an integral operator acting at the function depending on the transformed variables $\rho_{r}^{\prime}, \rho_{s}^{\prime}$

Let us consider now eq. (3) in the simple case of the three gluon system $(n=3)$ with odderon quantum numbers $\left(P_{j}=C=-1\right)$. Here the colour structure of $\chi$ is factorized and we can substitute $\left(-T_{r}^{a} T_{s}^{a}\right)$ by $\frac{1}{2} N=\frac{3}{2}$. After that we obtain the following equation for $\chi^{r}$ in eq. (14):

$\omega^{\prime} \chi^{r}=\frac{g^{2}}{8 \pi^{2}} \frac{3}{2} \sum_{1 \leqslant t<s \leqslant 3} k\left(M_{t s}^{2}\right) \chi^{r}, \quad \omega=\omega^{\prime}+\omega^{\prime \prime}$.

By putting here the conformally covariant and symmetric ansatz for a state $\chi(-8)$ with its conformal weight equal to $m$ $\chi^{\prime}=\left(\frac{\rho_{12} \rho_{13} \rho_{23}}{\rho_{10}^{2} \rho_{20}^{2} \rho_{30}^{2}}\right)^{m / 3} f^{r}(\chi), \quad x=\frac{\rho_{12} \rho_{30}}{\rho_{13} \rho_{20}}$,

we can construct the one dimensional equation for the function $f^{r}(x)$. The numerical solution of this equation will be published later [8].

An approximate method of solving eqs. (3), (17) was suggested in ref. [5]. It consists in using a "diffusion approximation" for $K(4)$ :

$K\left(\rho_{r}, \rho_{s}\right) \simeq 8 \ln 2+14 \zeta(3)\left(M_{r s}^{2}+M_{r s}^{* 2}+\frac{1}{2}\right)$,

which corresponds to expanding $k\left(M^{2}\right)(5)$ in a series near the point $M^{2}=-\frac{1}{4}$. In this approximation eq. (3) is simplified:

$$
\begin{aligned}
-\omega \chi & =H \chi, \\
H & =\frac{g^{2}}{8 \pi^{2}}\left(-[8 \ln 2+7 \zeta(3)] \frac{n N}{2}\right. \\
& \left.+14 \zeta(3)\left[\left(T_{r}^{a} M_{r}^{\mu}\right)^{2}+\left(T_{r}^{a} M_{r}^{* \mu}\right)^{2}\right]\right) .
\end{aligned}
$$

Here the summation over $a, \mu$ and $r$ is implied. $H$ commutes with the operators $\sum_{r=1}^{n} T_{r}^{b} M_{r}^{\nu}, \sum_{r=1}^{n} T_{r}^{b}$, $\sum_{r=1}^{n} M_{r}^{\nu}$ which are generating elements of an algebra. Therefore, $\omega$ can be calculated in terms of the highest weight for each representation of the algebra. These representations are infinitely degenerate because $H$ commutes with the operators $\left(\sum_{r} T_{r}^{h} M_{r}^{\nu}\right)^{2}$, $\left(\sum_{r} T_{r}^{b}\right)^{2},\left(\sum_{r} M_{r}^{\nu}\right)^{2}$ for all subsets $\tilde{r}$ of the set $r=1$, $2, \ldots, n$.

As is seen from eq. (20), in this model the position $\omega$ of the leading singularity of the partial waves grows linearly with the number $n$ of reggeized gluons in accordance with the pole approximation (13), which is apparently a general phenomenon. Nevertheless, the "diffusion approximation" is bad from the theoretical point of view because the operator on the RHS of eq. (19) in contrast with the exact one (4) is not restricted from above for states with a large conformal spin $n_{r s}$. Therefore, the odderon intercept obtained in ref. [5] with the use of this approximation is not reliable. One of the possible ways to correct this defect is to put some constraints on the solution of eq. (20) to suppress the contribution of the states with nonzero values of $n_{r s}$.

In conclusion, it is necessary to stress that contributions of diagrams with many reggeized gluons are 
very important for constructing the QCD scattering amplitudes at high energies satisfying general requirements of the $s$ - and $t$-channel unitarity.

I thank the Institute of the Nuclear Physics (Orsay) and the DESY Theory Division for their kind hospitality during my stay. I express my gratitude to B. Nicolescu, P. Gouron and J. Bartels for helpful discussions.

\section{References}

[1] E.A. Kuraev, L.N. Lipatov and V.S. Fadin, Sov. Phys. JETP 44 (1976) 433; 45 (1977) 199.
[2] V.S. Fadin and L.N. Lipatov, Institute of Nuclear Physics, Novosibirsk, preprint 89-13 (1989).

[3] Ya.Ya. Balitskii and L.N. Lipatov, Sov. J. Nucl. Phys. 28 (1978) 822.

[4] L. Kwiecinski and M. Praszalowicz, Phys. Lett. B 94 (1980) 413;

T. Jaroszewicz and K. Kwiecinski, Z. Phys. C 12 (1982) 167. [5] L.N. Lipatov, Sov. Phys. JETP 63 (5) (1986) 904.

[6] L.N. Lipatov, in: Perturbative QCD, ed. A.H. Mueller, Advanced Series in Directions in High Energy Physics (World Scientific, Singapore, 1989).

[7] A.A. Belavin, A.M. Polyakov and A.B. Zamolodchikov, Nucl. Phys. B 241 ( 1984 ) 333.

[8] P. Gouron, L. Lipatov and B. Nicolescu, work in progress. 